\title{
Pressure Pulsations of the Blade Region in S-Shaped Shaft-Extension Tubular Pumping System
}

\author{
Fan Yang and Chao Liu \\ School of Hydraulic, Energy and Power Engineering, Yangzhou University, Jiangsu, China \\ Correspondence should be addressed to Fan Yang; sqyzyangfan@126.com
}

Received 19 April 2014; Revised 4 June 2014; Accepted 12 June 2014; Published 6 July 2014

Academic Editor: L. W. Zhang

Copyright ( 2014 F. Yang and C. Liu. This is an open access article distributed under the Creative Commons Attribution License, which permits unrestricted use, distribution, and reproduction in any medium, provided the original work is properly cited.

\begin{abstract}
The three-dimensional unsteady numerical method is applied to investigate the pressure fluctuation of the S-shaft extension pumping system. Some monitor pointers are set at the key positions of blade region to capture the changing rules of the pressure pulsations, from which the data are collected through time-domain and spectrum analysis. The predicted head and efficiency were validated by the comparison with the tested results, and the comparison result shows that the unsteady flow characteristic of pumping system can be simulated by this method. The pulsating amplitude decreases gradually from blade tip to hub, under the condition of large flow rate for both the inlet and outlet of the impeller, in the way different from the smaller flow rate condition. Through adjusting the impeller rotation speed and the number of blades, the dominant frequency can be controlled. For the same monitor point at the impeller, as the flow coefficient is becoming larger, the pulsating amplitude decreases, in which case, it decreases firstly and then increases between the impeller and the guide vane, and the pulsating amplitude is smallest on the high efficiency condition. The rotation speed of impeller has little effect on the dominant frequency at the outlet of guide vane.
\end{abstract}

\section{Introduction}

Hydraulic model, composed of impeller and guide vane, plays an important role for high performance of hydraulic machinery system, of which the safety and efficiency depend on the hydrodynamic characteristics. The model pressure pulsation of interior flow is very difficult to be measured by traditional experimental equipment. Today, the pressure is often analyzed by numerical simulation. Wang et al. in [1] studied the unsteady flow feature in axial-flow pump based on the Reynolds-averaged Navier-Stokes method and the large eddy simulation method. Shi et al. in [2] applied the standard turbulent $k-\varepsilon$ model and SIMPLEC algorithm to investigate the pressure fluctuation induced by the rotorstator interactions of the mixed-flow pump. Wu et al. in [3] used the Reynolds-averaged Navier-Stokes equations coupled with the RNG $k-\varepsilon$ turbulent flow of the whole flow passage of a prototype Kaplan turbine. Shi et al. in [4] studied the dynamic pressure gradient model of axial piston and parameters optimization for stabilizing the pressure gradient in pressure rising and control the pressure of piston chamber.
Liu et al. in [5] studied characteristics of pressure pulsation of pump-turbines with misaligned guide vanes based on CFD commercial software. And some other domestic scholars have focused on the study of the pressure pulsation of interior flow in centrifugal pump by numerical simulation and test in [6$10]$.

In china, research on pressure pulsation of pumping system with low head has become a hotspot. Wang et al. in [11] analyzed the impeller elevation, unsteady flow, hydraulic thrust, and the zero-head flow of the slanted axial pumping system, using fluent software. Zhu et al. in [12] studied pressure pulsation of tubular pump internal pressure pulsation based on large eddy simulation data. Yang and Liu in [13] studied the characteristics of pressure pulsation of mixedflow pumping system, for irrigation and drainage, through performance test. Given the fact that pressure pulsation of blade region in shaft-extension tubular pumping system is rarely studied domestically, in this paper, taking the S-shaped shaft-extension as the research object, three-dimensional flow field of pumping system has been simulated by the programming of the commercial code ANSYS-CFX; it was 


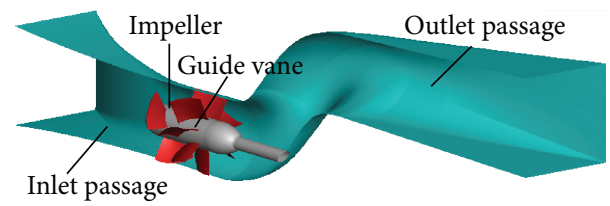

FIgURE 1: Perspective of S-shaped shaft-extension tubular pumping system.

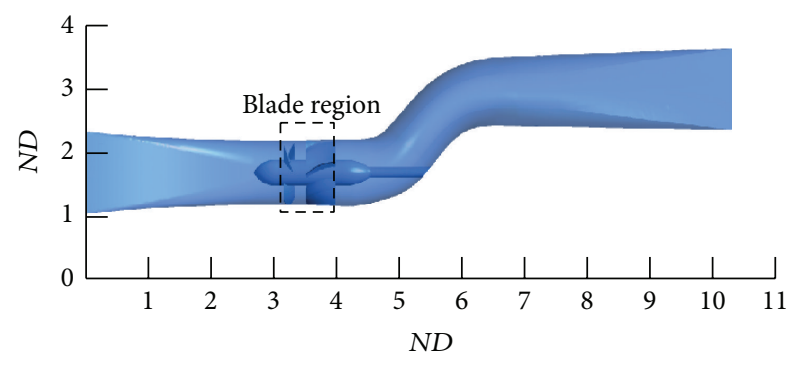

Figure 2: Blade region.

analyzed on three different operating conditions, offering a reference for safer and more stable operation of this type of pump.

\section{Pumping System Geometry and Numerical Method}

Figure 1 shows 3D geometric of S-shaped shaft tubular pumping system model, with specific speed $n_{s}=970$ and impeller diameter 300, which is used to simulate and measure its entire flow field. The impeller has 3 blades and the stator has 7 blades. The rotating speed $n$ is $1450 \mathrm{r} / \mathrm{min}$. The average tip clearance of impeller is $0.2 \mathrm{~mm}$ at blade angle $0^{\circ}$, which are the same as those of the experimental model. The blade region is defined as Figure 2, $N$ is integer, and $D$ is impeller diameter.

The grid quality in the impeller and guide vane will affect the accuracy of pumping system simulation. Accordingly, $\mathrm{H} / \mathrm{J} / \mathrm{L}$-type topology structure is applied to the impeller, and H-type topology structure is applied to the guide vane. O-type grid is used at the blade surface region to control boundary layer distribution. Grid refinement is applied to impeller with 18 nodes in the tip clearance. A structured grid system is constructed in the computational domain. Surface grid of every flow component is shown as Figure 3.

Prior to step for analysis of pumping system, in order to determine the optimal number of grids, a preliminary grid dependency test with numbers of nodes ranging from 1000000 to 2200000 is carried out. The computations have shown that grid convergence has been obtained for the performance of the S-shaped shaft-extension tubular pumping system at $K_{Q}=0.460$, as shown in Figure 4 . With the increase of grid numbers, the head coefficient $K_{H}$ and efficiency $\eta$ change greatly at first then change very little gradually. Finally, the appropriate number of grid numbers for the simulation was determined by the preliminary simulation results. In the present study, grid number 1516416 is selected as the optimum number of grids.

The commercial URANSE-CFD-Solver ANSYS CFX 14.0 was used. ANSYS CFX uses the element based finite volume method and an algebraic multigrid approach. The timestep for the transient calculation (eight impeller revolutions) represented 1 of rotation. So, 2880 timesteps are calculated. Every timestep consisted of 8 to 22 inner coefficient loops. The turbulence effects are modeled by the RNG $k$ - $\varepsilon$ turbulence model. The RNG model provides a way to account for the effects of swirl or rotation by modifying the turbulent viscosity appropriately. A uniform axial velocity based on the massflowrate is specific at the inlet for each computation run, and the total pressure at the outlet is set to $1.2 \mathrm{~atm}$. The transient rotor-stator method is used for interface condition between the rotating impeller and stationary diffuser. Scalable wall functions are used to simulate the boundary layers. Adiabatic and hydraulically smooth walls with no slip condition were considered at solid boundaries. Periodic boundaries are set at the blade passage interfaces.

\section{Performance Prediction and Results Comparison}

3.1. Test Device and Method. Physical model of pumping system is tested in the Hydrodynamic Engineering Laboratory of Jiangsu Province. Physical model is shown in Figure 5. The sketch of the complete test rig is shown in Figure 6. Pumping system head is measured by differential pressure transmitter EJA110A, and measuring sections are chosen in the tanks with the inlet and outlet passages for concluding hydraulic loss of inlet and outlet sections. Torque is measured by ZJ type torque meter, which is transmitted by pump shaft. Flowrate is measured by E-mag type electromagnetic flowmeter.

3.2. Results Comparison. According to test data, the highest efficiency of pumping system is $83.55 \%$ at blade angle $\theta=-2^{\circ}$, with flowrate coefficient $K_{Q}$ of 0.443 and head coefficient $K_{H}$ of 0.828 . With flowrate coefficient $K_{\mathrm{Q}}$ of 0.478 and head coefficient $K_{H}$ of 0.887 at blade angle $\theta=0^{\circ}$, the efficiency $\eta$ is $82.57 \%$. Dynamic characteristics prediction method is shown in

$$
\begin{array}{rlrl}
\bar{Q} & =\frac{\sum_{i=1}^{M} \dot{Q}_{i}}{M}, & \bar{H}=\frac{\sum_{i=1}^{M} \dot{H}_{i}}{M}, & \bar{P}=\frac{\sum_{i=1}^{M} \dot{P}_{i}}{M}, \\
\bar{\eta}=\frac{\rho g \bar{Q} \bar{H}}{M}, & K_{Q}=\frac{\bar{Q}}{n D^{3}}, & K_{H}=\frac{g \bar{H}}{n^{2} D^{2}} .
\end{array}
$$

The prediction data agree with the experimental head and efficiency, as shown in Table 1 . From the predicted results, the maximal deviation of $K_{H}$ is $3.15 \%$, and the maximal deviation of $\eta$ is $3.07 \%$ in the unsteady simulation. The simulation results agree with test data very well.

To verify the simulation with RNG $k$ - $\varepsilon$ turbulence model and Reynolds time-averaged equation, a comparison between the simulation and experimental results is made, as shown in Figure 7. The position concerning the pressure fluctuations is a measuring point on the outlet section of the inlet passage. 


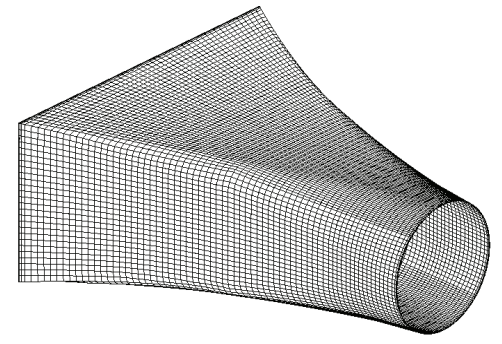

(a) Inlet passage

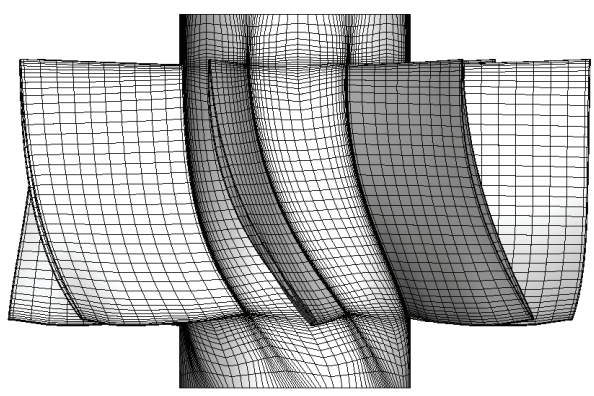

(c) Guide vane

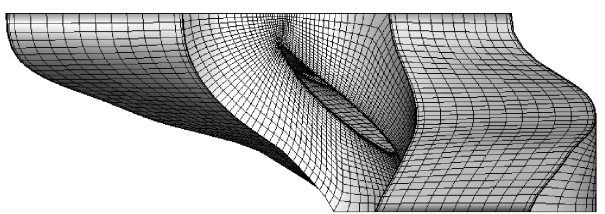

(b) Impeller

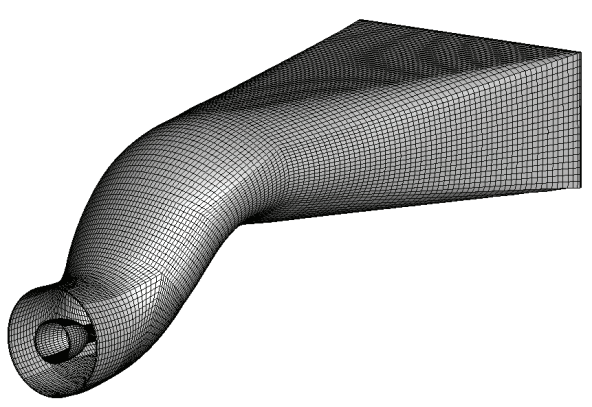

(d) Outlet passage

FIgURE 3: Computational grids.

TABLE 1: Experimental and predicted results.

\begin{tabular}{lccr}
\hline Flow coefficient $K_{Q}$ & Test results $K_{H}$ & Unsteady prediction $K_{H}$ & Deviation/\% \\
\hline 0.368 & 1.249 & 1.222 & 2.16 \\
0.402 & 1.143 & 1.107 & 3.15 \\
0.460 & 0.945 & 0.964 & 2.01 \\
0.508 & 0.761 & 0.774 & 0.71 \\
0.552 & 0.529 & Unsteady prediction $\eta / \%$ & 0.95 \\
\hline Flow coefficient $K_{Q}$ & Test results $\eta / \%$ & 71.10 & 1.69 \\
\hline 0.368 & 69.92 & 74.87 & 1.12 \\
0.402 & 75.72 & 81.57 & 0.11 \\
0.460 & 81.66 & 80.47 & 0.94 \\
0.508 & 81.23 & 72.17 & 3.07 \\
0.552 & 70.02 & Deviation/\% \\
\hline
\end{tabular}

The numerically calculated pressure fluctuation follows the trend very well as compared to the experimental ones. The dominant frequencies and their amplitudes for simulation and experimental results are $72.5 \mathrm{~Hz}, 0.0530,72.5 \mathrm{~Hz}, 0.0491$, respectively. This indicated that the simulation with RNG $k$ $\varepsilon$ turbulence model and Reynolds time-averaged equation could be used to predict the pressure fluctuation in the pumping system in the view of engineering.

\section{Pressure Pulsation Results and Discussions}

To analyze pressure pulsation of blade region, 26 monitoring points are made in the blade region to monitor it and the developing trend thereof. Four groups of measuring points are shown in Figure 8 , distributed as 8 measuring points
(P01 P08) in the impeller inlet, 4 measuring points (P09 $\mathrm{P} 16)$ between the impeller and guide vane, 4 measuring points (P17 P20) inside the guide vane, and 6 measuring points (P21 P26) in the guide vane outlet.

In the analysis of pressure pulsation of blade region, mathematical statistics and spectrum analysis are applied. Hydraulic pressure process is assumed to be a stationary stochastic process for spectrum analysis, and the irregular pressure pulsation is decomposed into many superimposed results of simple harmonic wave, including different amplitudes, frequencies, and phases. Spectrum analysis is adopted by many scholars for pressure pulsation analysis, for it can overcome the shortage of randomness of mathematical statics; in this paper it is used in the blade region, whereby pressure coefficient $C_{p}$ is defined in [2], and fast Fourier 


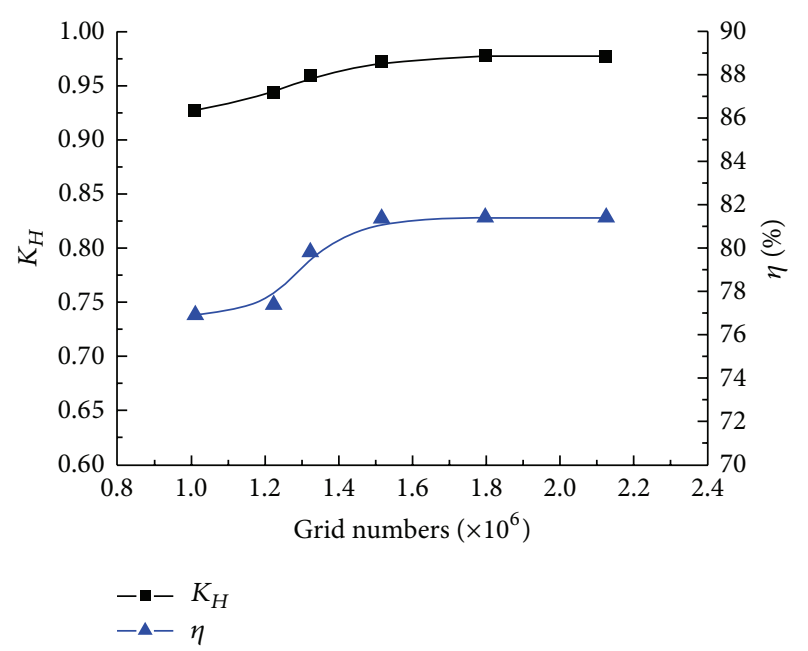

Figure 4: Verification of grid independence to prediction of performances.

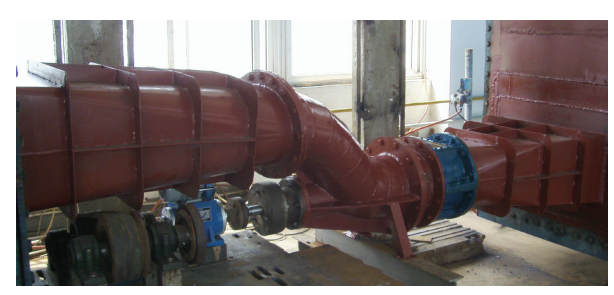

FIgURe 5: Physical model.

transform (FFT) is applied to show the unsteady pressure features in frequency domain based on the pressure time domain data results of unsteady simulation.

In this calculation model, pressure of pumping system acquired can meet the periodic requirements after 1440 timesteps, whereby the 7th rotating cycle is chosen for the analysis of the unsteady characteristics.

4.1. Pressure Pulsation of Impeller Inlet. The pulsation spectrums of 8 monitoring points in 3 different operating conditions for pumping system in 3 different operating conditions are shown in Figure 9. Pulsation amplitude increases gradually from the hub to the tip in different operating conditions. The pressure coefficient magnitude of monitoring point $\mathrm{P} 01$ is 1.381 times that of monitoring point P04, and the pressure coefficient magnitude of monitoring point P08 is 1.379 times that of monitoring point P05 in small flowrate condition $K_{Q}=0.368$. The pressure coefficient magnitude of monitoring point $\mathrm{P} 01$ is 1.405 times that of monitoring point P04, and the pressure coefficient magnitude of monitoring point $\mathrm{P} 08$ is 1.410 times that of monitoring point $\mathrm{P} 05$ in high efficiency condition $K_{Q}=0.460$. The pressure coefficient magnitude of monitoring point $\mathrm{P} 01$ is 1.348 times that of monitoring point $\mathrm{P} 04$, and the pressure coefficient magnitude of monitoring point P08 is 1.352 times that of monitoring point P05 in large flowrate condition $K_{Q}=0.552$. It is obvious that the trend of pressure amplitude is the same in two sides of impeller inlet. The velocity flow field of impeller

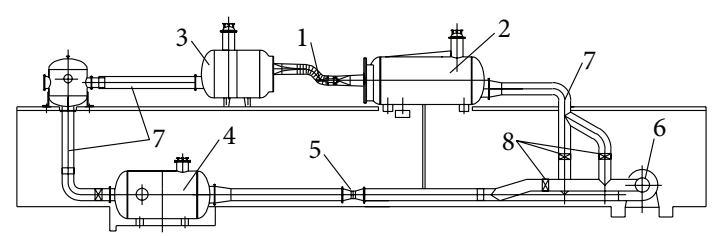

Figure 6: Sketch of test rig. (1) Pumping system model, (2) inlet water tank, (3) outlet water tank, (4) surge tank, (5) electromagnetic flowmeter, (6) auxiliary pump, (7) pipeline, and (8) valve.

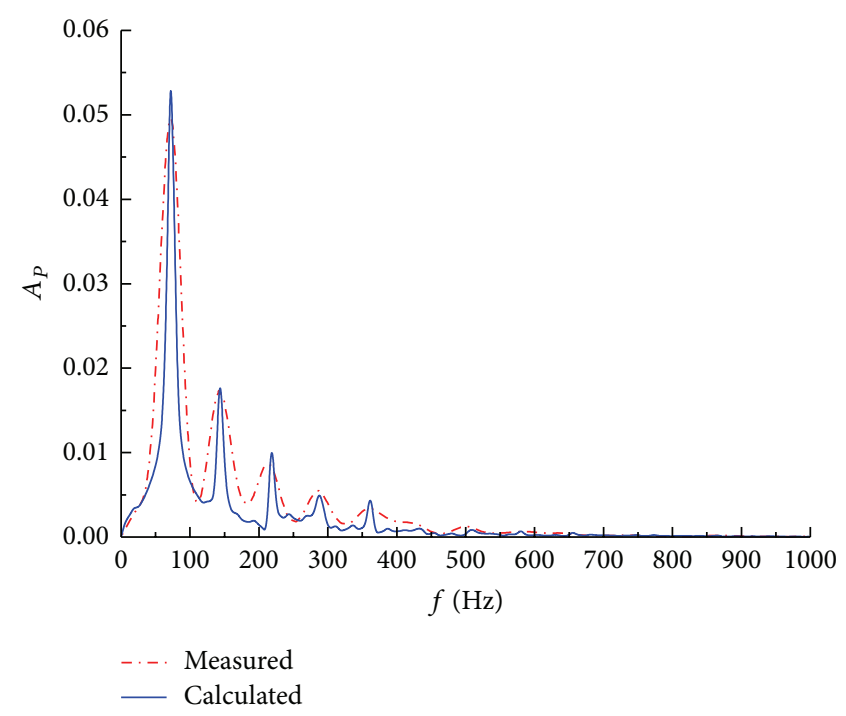

Figure 7: Pressure fluctuations for a position in inlet passage.

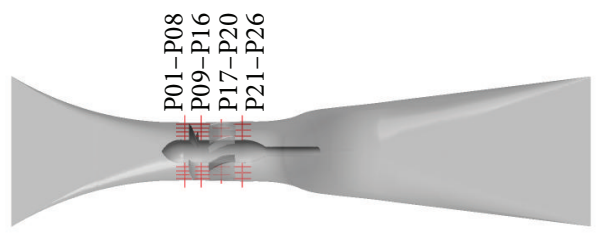

FIGURE 8: Pressure monitoring points.

inlet is symmetric distribution. The rotating of impeller has little effects on the distribution of velocity components at the impeller inlet. For the same monitoring points, pressure pulsation amplitude decreases gradually with the increase of flowrate. The dominant frequency of every monitoring point is $72.5 \mathrm{~Hz}$ in impeller inlet, which is equal to the blade passing frequency. Pressure pulsation of impeller inlet is mainly influenced by rotating speed of impeller and blade numbers.

4.2. Pressure Pulsation between Impeller Outlet and Guide Vane Inlet. The pulsation spectrums of P09 P16 are shown in Figure 10. In small flowrate operating condition $K_{Q}=$ 0.368 , the relative difference of pressure pulsation is higher for symmetric point, the minimum relative difference is $7.47 \%$, and the maximum relative difference is $19.35 \%$. In high efficiency operating condition $K_{\mathrm{Q}}=0.460$, the minimum relative difference of symmetric points is $11.76 \%$, and the 


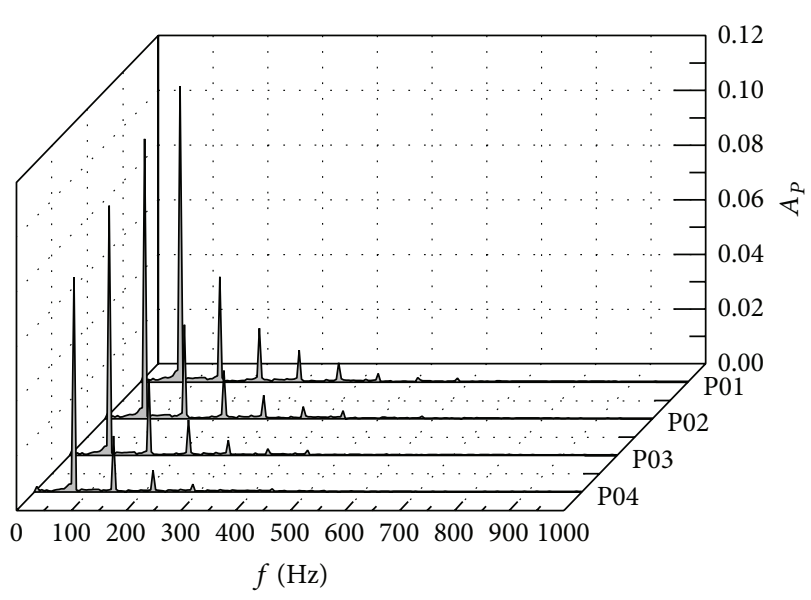

(a) Small flowrate condition $\left(K_{Q}=0.368\right)$

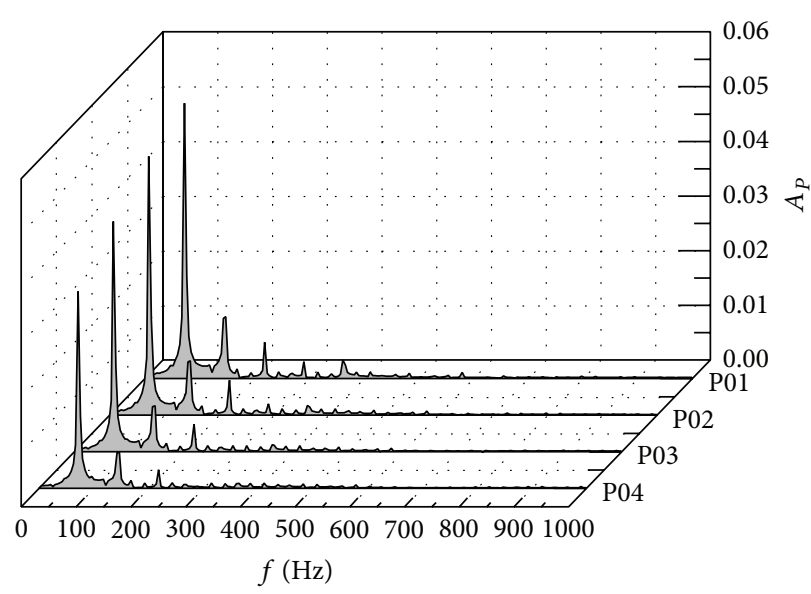

(c) Large flowrate condition $\left(K_{Q}=0.552\right)$

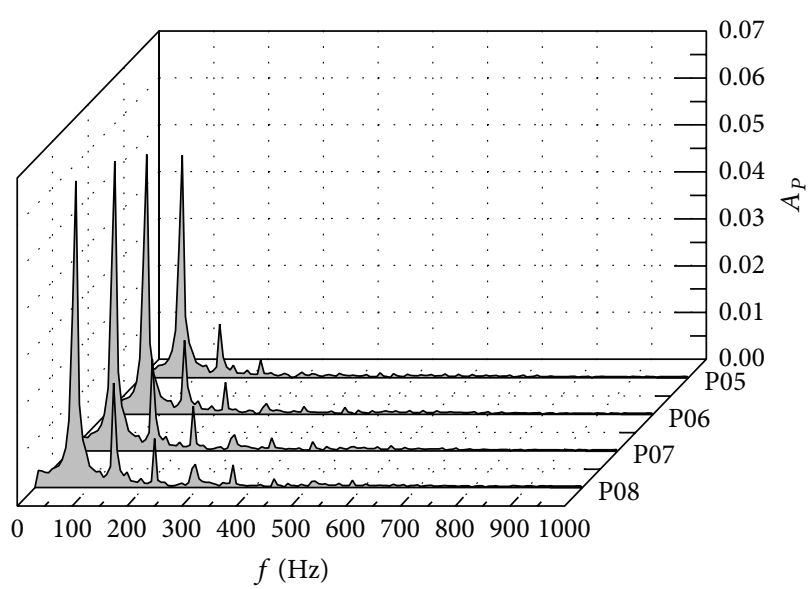

(e) High efficiency condition $\left(K_{Q}=0.460\right)$

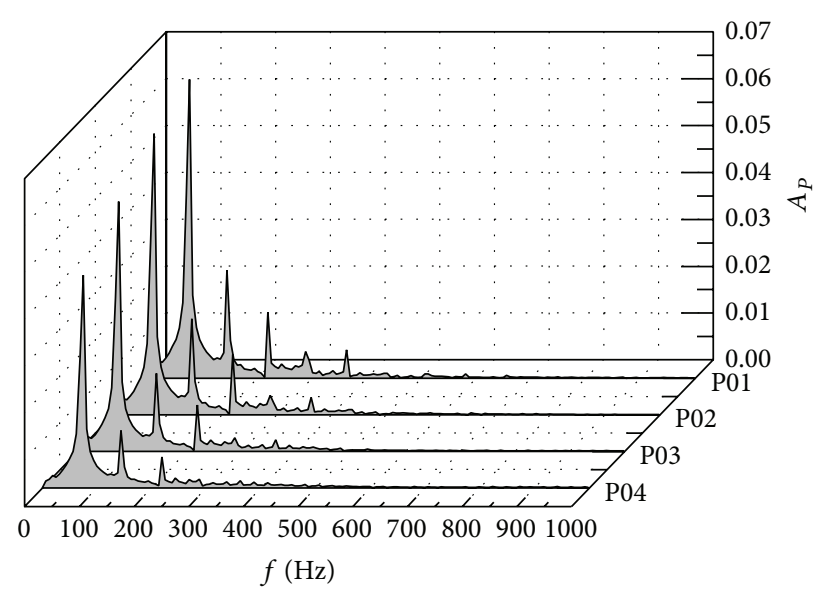

(b) High efficiency condition $\left(K_{Q}=0.460\right)$

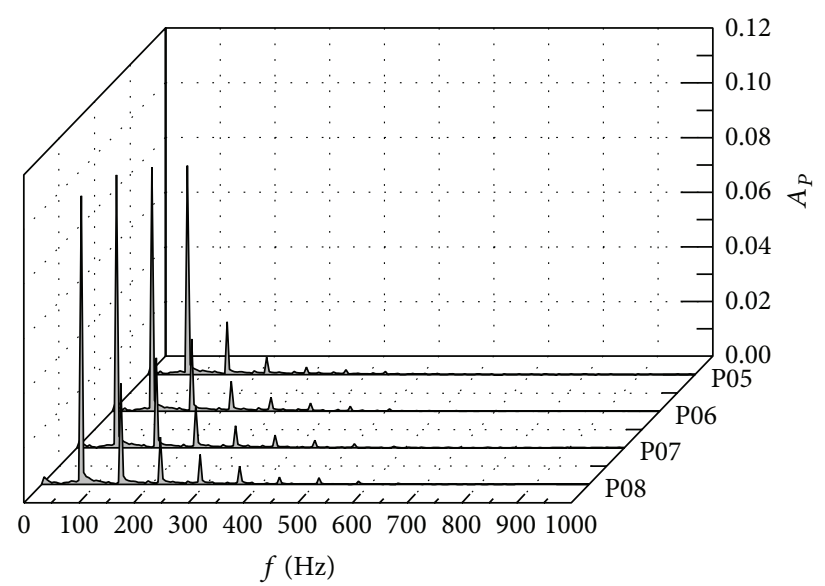

(d) Small flowrate condition $\left(K_{Q}=0.368\right)$

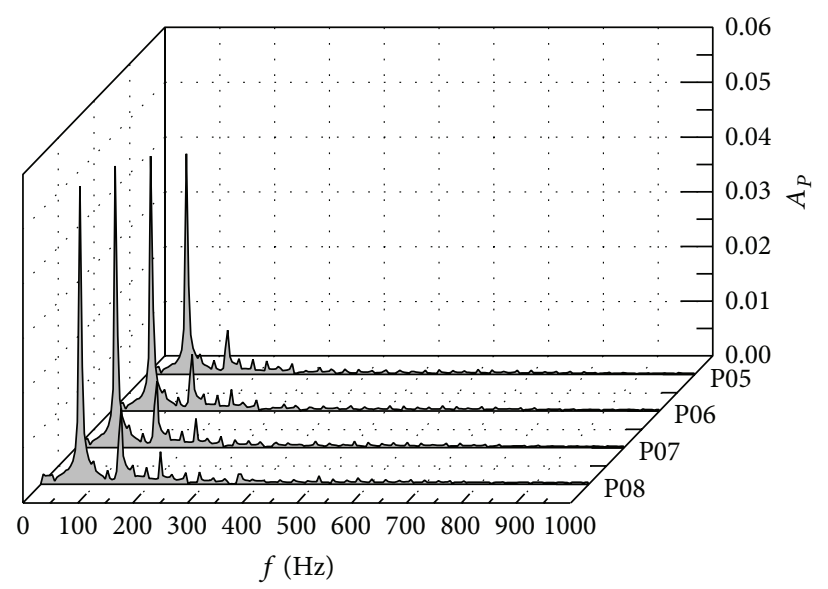

(f) Large flowrate condition $\left(K_{Q}=0.552\right)$

Figure 9: The pulsation spectrums of P01 P08 at impeller inlet under different operating conditions.

maximum relative difference is $22.39 \%$. Compared with small flowrate and high efficiency operating conditions, the relative difference of symmetric points is larger in large flowrate operating condition $K_{Q}=0.552$; the minimum relative value is $11.81 \%$, and the maximum relative value is $14.56 \%$. The analytic results show that the velocity distribution of impeller outlet is obviously asymmetric in $K_{Q}=0.368$, which is mainly affected by velocity circulation of impeller outlet in this operating condition.

In $K_{\mathrm{Q}}=0.368$, the pulsation amplitude decreases gradually from P09 to P12, the pulsation amplitude increases firstly then decreases from P16 to P13. The pulsation amplitude 


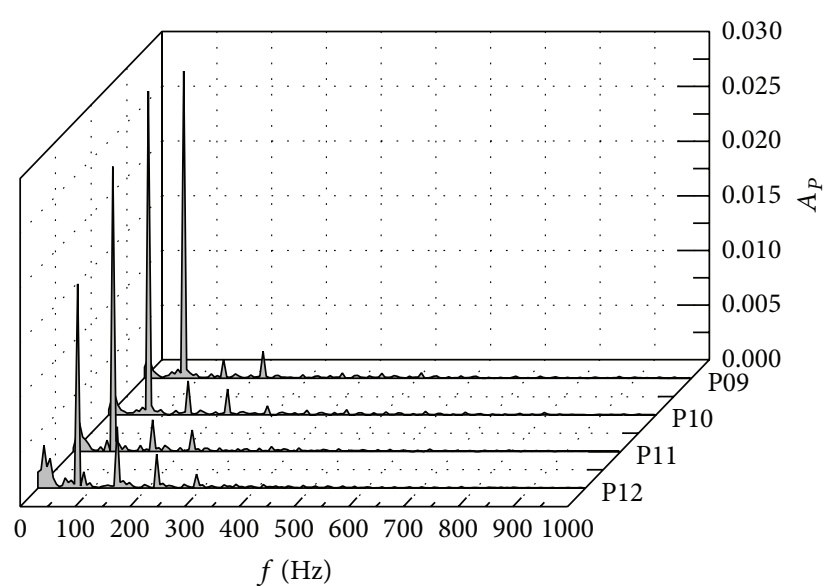

(a) Small flowrate condition $\left(K_{\mathrm{Q}}=0.368\right)$

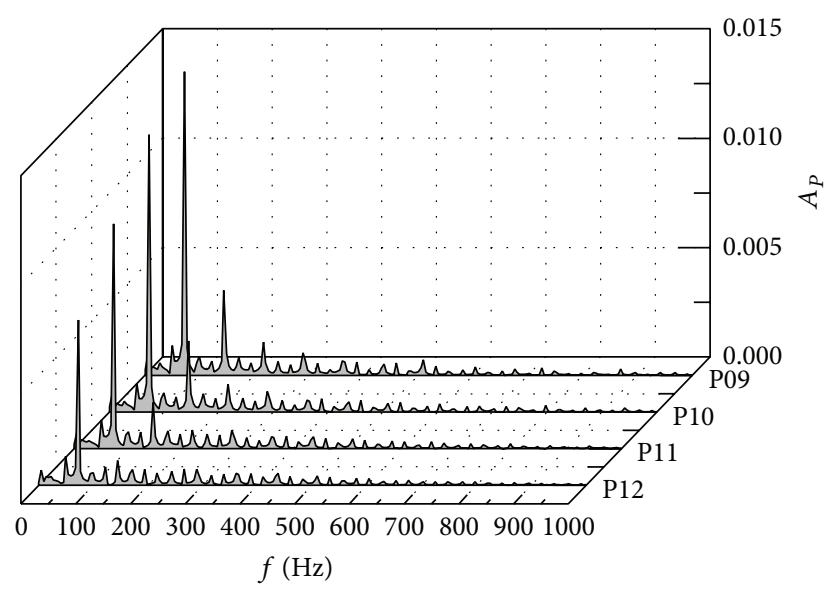

(c) Large flowrate condition $\left(K_{Q}=0.552\right)$

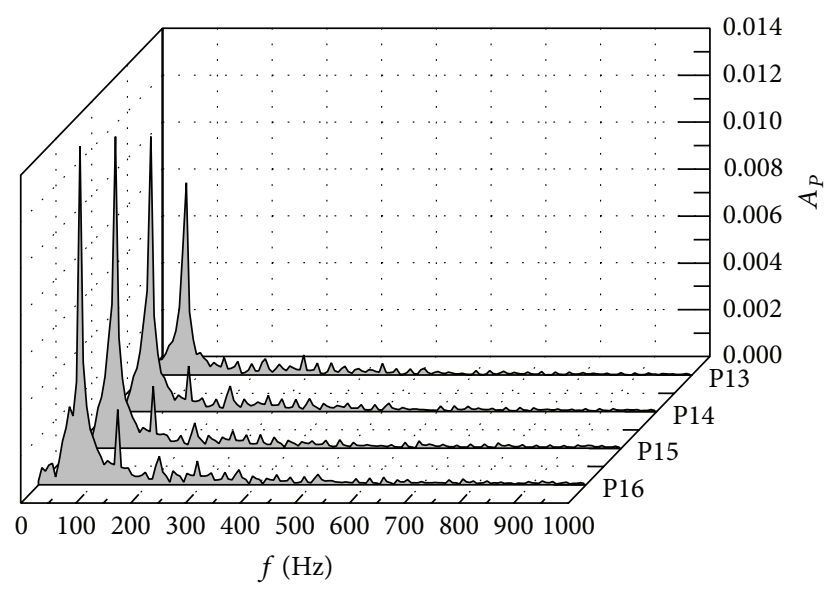

(e) High efficiency condition $\left(K_{Q}=0.460\right)$

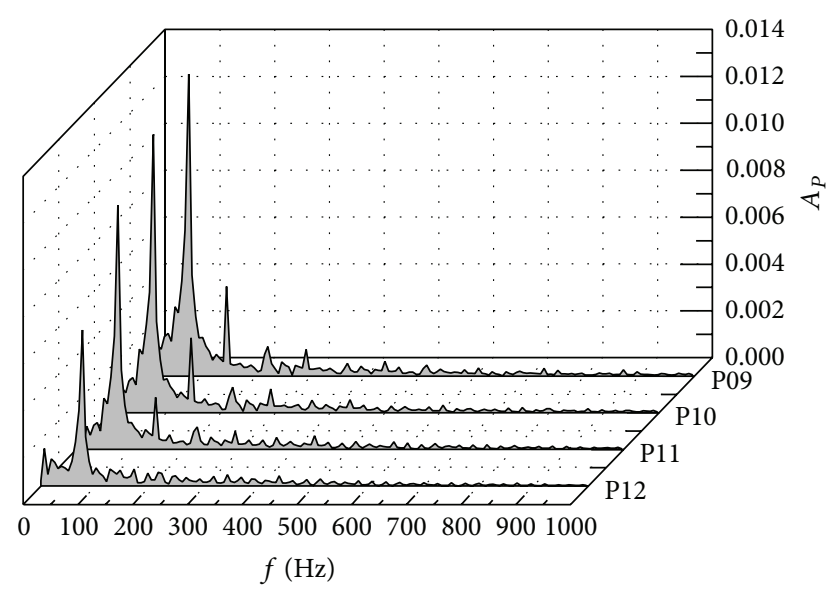

(b) High efficiency condition $\left(K_{Q}=0.460\right)$

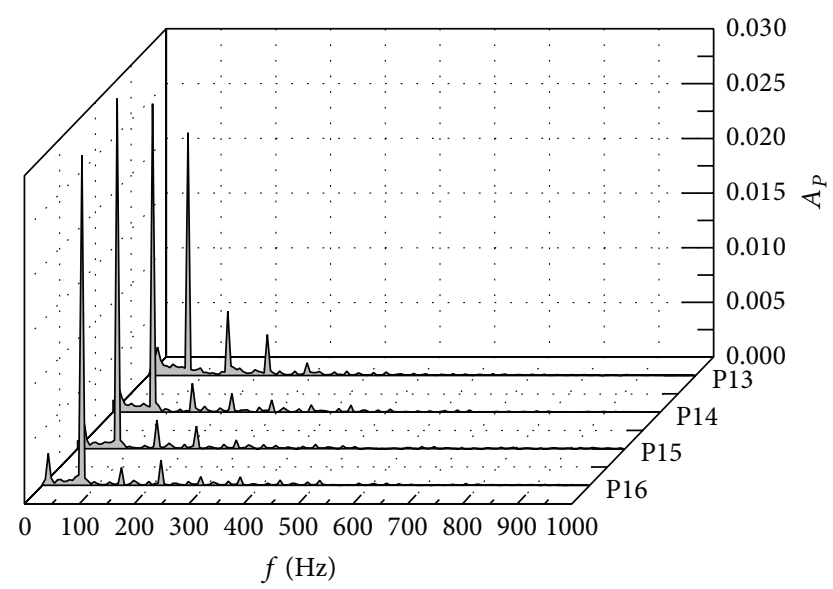

(d) Small flowrate condition $\left(K_{Q}=0.368\right)$

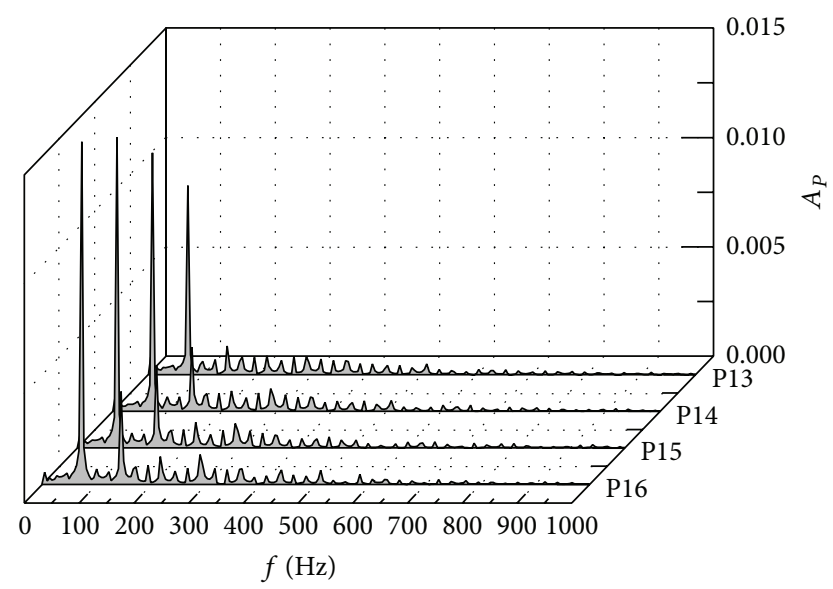

(f) Large flowrate condition $\left(K_{Q}=0.552\right)$

Figure 10: The pulsation spectrums of P09 P16 between impeller and guide vane.

decreases gradually from blade tip to hub for monitoring points of the same side in $K_{Q}=0.460$ and $K_{Q}=0.552$. The internal flow of impeller and guide vane is more complex in small flowrate operating condition, because the characteristic of rotor-stator interaction is very significant. In order to decrease the pressure pulsation between impeller and guide vane, people should pay attention to select and regulate the pumping system operation conditions. The dominant frequency of P09 P16 is $72.5 \mathrm{~Hz}$, which is affected by both rotation frequency and blade number. The pulsation amplitude of impeller inlet is larger than that between impeller and guide vane in the same operating condition. 


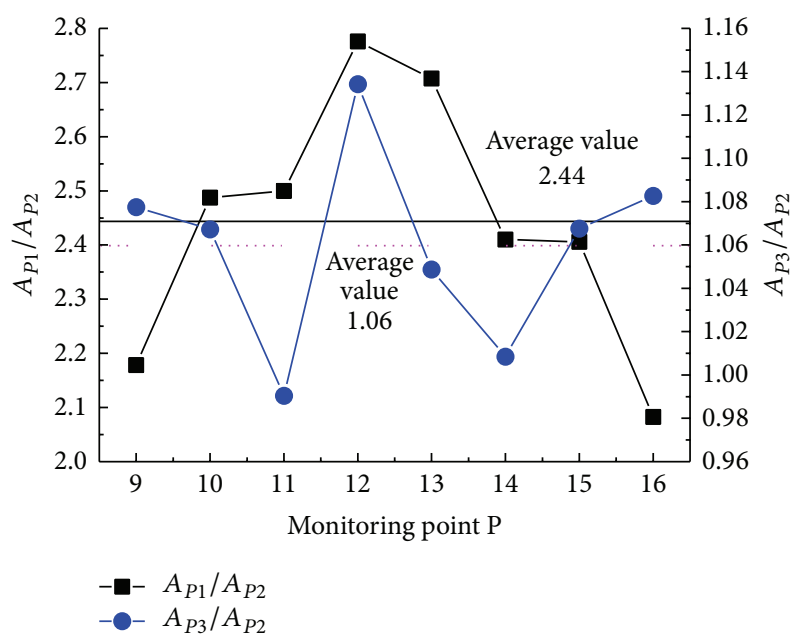

FigURE 11: The ratio of pressure coefficient amplitude under different conditions.

To further describe change of the pulsation amplitude between impeller and guide vane on three operation conditions, the pressure coefficient amplitude is defined as $A_{\mathrm{P} 1}$ in $K_{Q}=0.368$, the pressure coefficient amplitude is defined as $A_{\mathrm{P} 2}$ in $K_{\mathrm{Q}}=0.460$, and the pressure coefficient amplitude is defined as $A_{\mathrm{P} 3}$ in $K_{\mathrm{Q}}=0.552$. The pressure coefficient amplitude $A_{\mathrm{P} 2}$ is used as reference for the same monitoring point in different operating conditions. The calculation results are shown in Figure 11.

In $K_{Q}=0.368$, the average value of pressure coefficient amplitude is 2.44 times that in $K_{Q}=0.460$ for every monitoring points, and every monitoring point is higher than that in high efficiency operating condition. In $K_{Q}=$ 0.552 , the average value of pressure coefficient amplitude is 1.06 times that in $K_{Q}=0.460$ for every monitoring point, and every monitoring point is higher than that in high efficiency operating condition except monitoring P09 and P16, while pulsation amplitude of P09 and P16 near blade tip is lower than that in high efficiency operating condition. The pulsation amplitude decreases firstly then increases for monitoring points between impeller and guide vane; in particular, the pulsation amplitude is minimum in high efficiency operating condition.

4.3. Pressure Pulsation of Guide Vane. The pressure pulsation time domain charts and pulsation spectrums of P17 P20 is shown in Figure 12. The results of spectrum analysis for P17 P20 are shown in Table 2, where F1 is dominant frequency and F2 is secondary dominant frequency. The flow of guide vane is far away from impeller outlet, but pressure pulsation of guide vane is affected by impeller rotating. The dominant or secondary frequency of every monitoring point is $72.5 \mathrm{~Hz}$, which is the same as the rotating frequency. Low frequency pulsation is main part of pressure pulsation frequency. The characteristics of pressure pulsation are not obvious, because the internal flow of guide vane is complex. With the flow into guide vane, pressure coefficient amplitude decreases gradually along the flow path, because guide vane recovers velocity circulation of impeller outlet and makes flow steady. The average pressure pulsation amplitudes in sections of P09 P16 and P17 P20 was taken for example. In $K_{Q}=0.368$, the average pressure pulsation amplitude decreases by $16.98 \%$ compared with section of P09 P16. The average pressure pulsation amplitude decreases by $65.40 \%$ in $K_{Q}=0.460$, and the average pressure pulsation amplitude decreases by $70.89 \%$ in $K_{Q}=0.552$. The velocity circulation of impeller outlet is recovered by guide vane in the high efficiency and large flowrate operating conditions.

4.4. Pressure Pulsation of Guide Vane Outlet. The dominant frequency and pulsation amplitude of P21 P26 are shown in Figure 13 in the three operating conditions of pumping system. In small flowrate coefficient $K_{Q}=0.368$, the dominant frequency of P21 P23 is 1.125 times that of rotating frequency, the dominant frequency of P24 P25 is 0.375 times that of rotating frequency, and the dominant frequency of P26 is 0.563 times that of rotating frequency. In high efficiency coefficient $K_{Q}=460$, the dominant frequency of P21 P23 is 0.563 times that of rotating frequency, the dominant frequency of P24 is 3 times that of rotating frequency, the dominant frequency of P25 is 0.75 times that of rotating frequency, and the dominant frequency of P26 is 0.188 times that of rotating frequency. In large flowrate coefficient $K_{Q}=$ 0.552 , the dominant frequency of P21 is 0.882 times that of rotating frequency, the dominant frequency of P22 P25 is 0.176 times that of rotating frequency, and the dominant frequency of $\mathrm{P} 26$ is 0.353 times that of rotating frequency. The rotating impeller and blade numbers have little influence on the pressure pulsation of guide vane outlet.

\section{Conclusion}

The unsteady turbulent flow in the pumping system was simulated based on software ANSYS CFX, and the pressure pulsations of different monitoring points were analyzed. Compared to the test performance results, the unsteady results are in agreement with unsteady prediction results, and the maximal error of unsteady prediction is only 3.15\%.

As the flowrate increases, the pressure pulsation amplitude of the same monitoring point decreases gradually in the impeller inlet, and it decreases gradually from blade tip to hub; it is mainly affected by impeller rotating in the impeller inlet, whereby the dominant frequency of this section is $72.5 \mathrm{~Hz}, 3$ times that of rotation frequency.

The pressure pulsation amplitude between impeller and guide vane decreases from blade tip to hub on the high efficiency and large flowrate conditions, while, on the small flowrate condition, the change law from blade tip to hub in the both sides of pump shaft axis is different; it decreases gradually from blade tip to hub in one side and increase earlier then decrease later in another side. The dominant frequency of pressure pulsation between impeller and guide vane is mainly affected by blade numbers and rotation frequency. When $K_{Q}=(0.368 \sim 0.552)$, the pressure pulsation amplitude decreases at the beginning then increases; it is minimum in the high efficiency area and greatest at the 


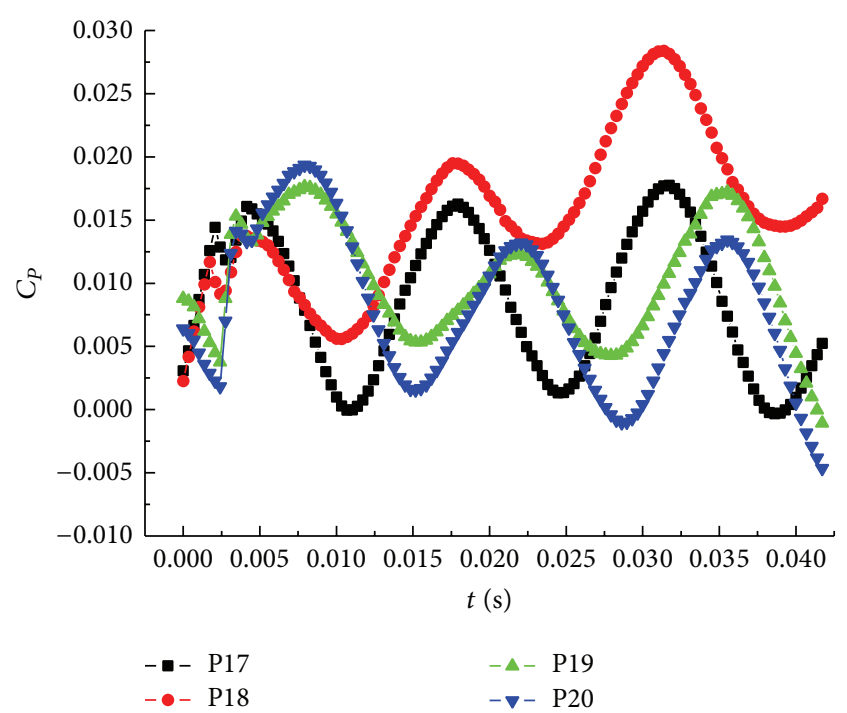

(a) Small flowrate condition $\left(K_{Q}=0.368\right)$

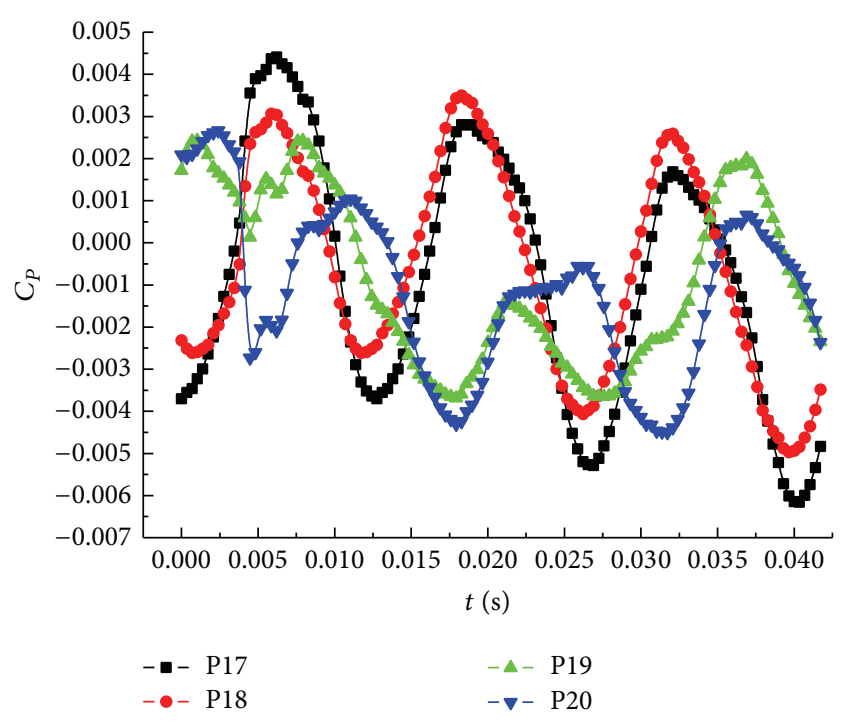

(c) Large flowrate condition $\left(K_{Q}=0.552\right)$

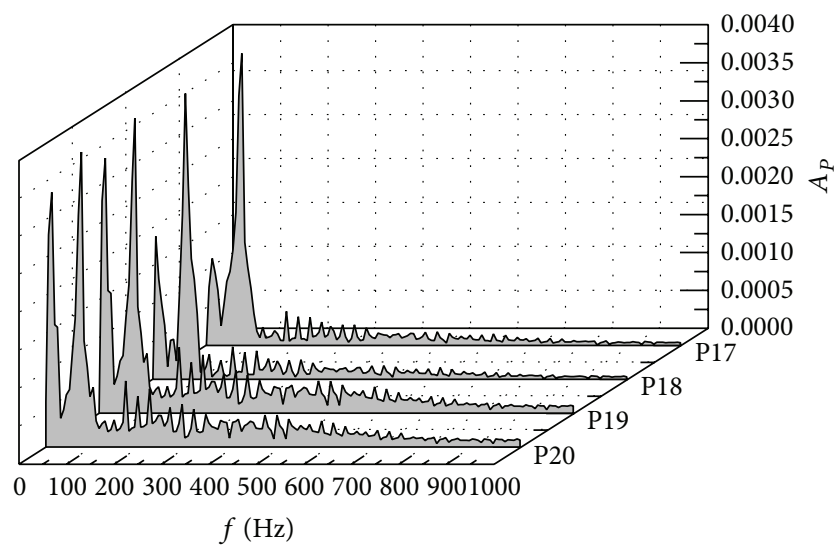

(e) High efficiency condition $\left(K_{Q}=0.460\right)$

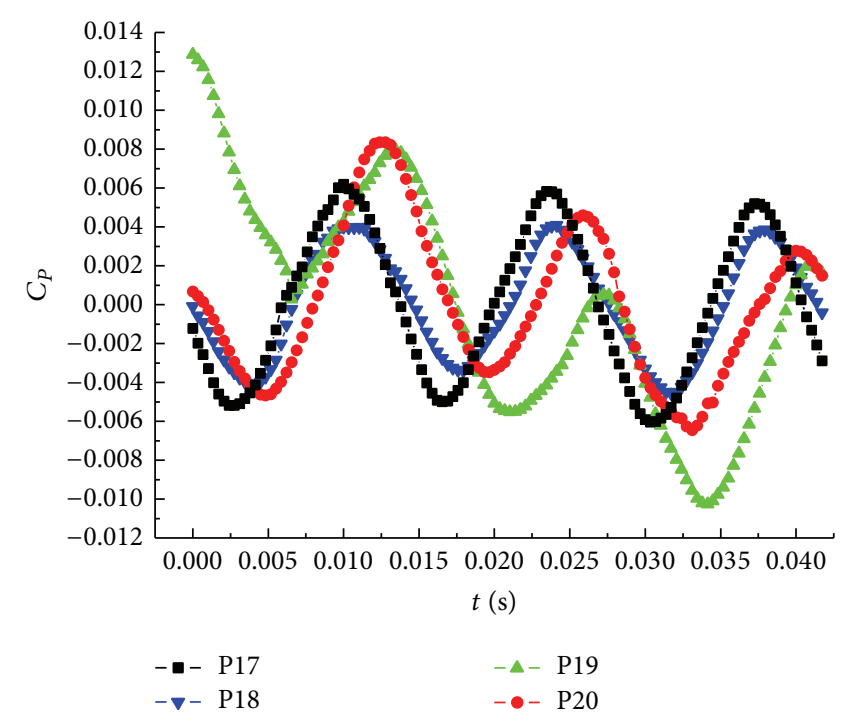

(b) High efficiency condition $\left(K_{Q}=0.460\right)$

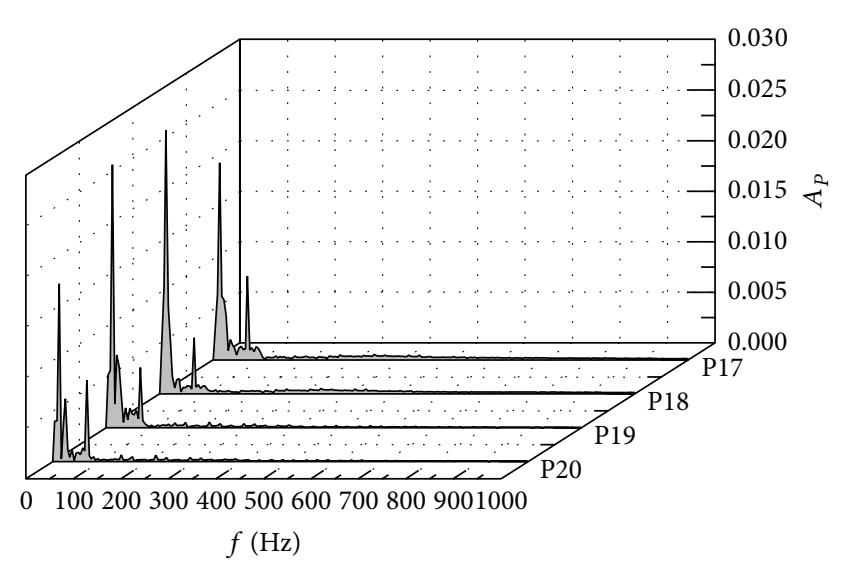

(d) Small flowrate condition $\left(K_{Q}=0.368\right)$

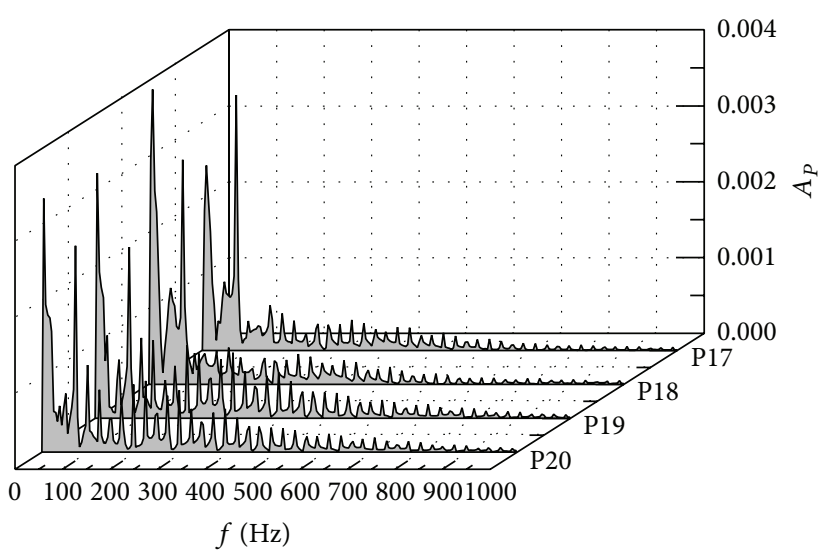

(f) Large flowrate condition $\left(K_{\mathrm{Q}}=0.552\right)$

FIGURE 12: Time domain and frequency spectra of pressure pulsations of P17 P20. 
TABLE 2: Frequency analysis of pressure pulsation of P17 P20.

\begin{tabular}{lccccccccccc}
\hline \multirow{2}{*}{ Flowrate coefficient $K_{Q}$} & \multirow{2}{*}{ Spectral parameter } & \multicolumn{2}{c}{ P17 } & \multicolumn{2}{c}{ P18 } & \multicolumn{2}{c}{ P19 } & P20 \\
& & F1 & F2 & F1 & F2 & F1 & F2 & F1 \\
\hline \multirow{2}{*}{0.368} & $f / H z$ & 13.59 & 72.50 & 13.59 & 72.50 & 13.59 & 72.50 & 13.59 & 72.50 \\
& $A_{P}$ & 0.0195 & 0.0083 & 0.261 & 0.0055 & 0.0260 & 0.0060 & 0.0176 & 0.0080 \\
0.460 & $f / H z$ & 72.50 & 66.46 & 66.46 & 72.50 & 72.50 & 12.08 & 72.50 & 12.08 \\
& $A_{P}$ & 0.0039 & 0.0033 & 0.0038 & 0.0026 & 0.0039 & 0.0034 & 0.0039 & 0.0034 \\
0.552 & $f / H z$ & 72.50 & 8.53 & 72.50 & 8.53 & 4.26 & 72.50 & 4.26 & 72.50 \\
& $A_{P}$ & 0.0034 & 0.0024 & 0.0039 & 0.0030 & 0.0032 & 0.0023 & 0.0033 & 0.0027 \\
\hline
\end{tabular}

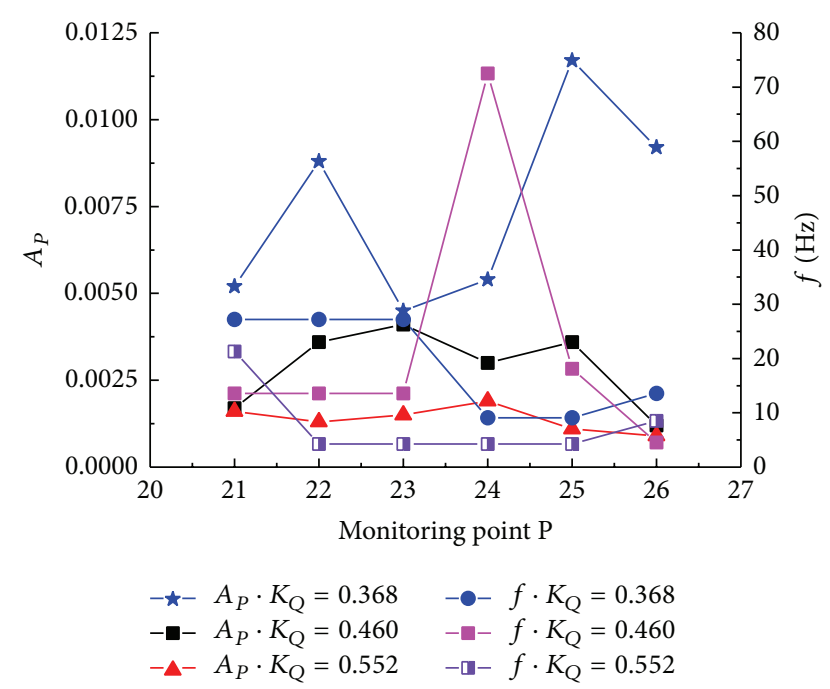

FIGURE 13: Frequency spectra and pressure coefficient amplitude of P21 P26.

impeller inlet, and its average value decreases sharply from the impeller inlet to the guide vane outlet.

The rotation impeller has little influence on the pressure pulsation of guide vane, when the dominant or secondary frequency is 3 times that of rotation frequency. The change of pressure pulsation in the guide vane is not prominent.

\section{Nomenclature}

$\bar{Q}: \quad$ Average flowrate

$\dot{Q}: \quad$ Static flowrate of every timestep

$M$ : Total number of timesteps for 1 rotating cycle

$\bar{H}$ : Average pumping system head

$\dot{H}$ : Static head of every timestep

$\bar{P}: \quad$ Average power

$\dot{P}_{i}:$ Static power of every timestep

$\bar{\eta}: \quad$ Average efficiency of pumping system

$\rho: \quad$ Fluid density

$g$ : Acceleration of gravity

$K_{\mathrm{Q}}$ : Flowrate coefficient

$n: \quad$ Rotation speed

$D$ : Diameter of impeller

$K_{H}$ : Head coefficient.

\section{Conflict of Interests}

The authors declare that there is no conflict of interests regarding the publication of this paper.

\section{Acknowledgments}

This research work was supported by the National Natural Science Foundation of China (Grant nos. 51279173 and 51376155) and the Natural Science Major Program of Jiangsu Provincial Colleges (Grant no. 11KJA570001). Support for construction/assembly of the facility was also provided by the Hydrodynamic Engineering Laboratory of Jiangsu Province.

\section{References}

[1] F.-J. Wang, L. Zhang, Y.-J. Li, and Z.-M. Zhang, "Some key issues of unsteady turbulent numerical simulation in axial-flow pump," Chinese Journal of Mechanical Engineering, vol. 117, no. 3, pp. 73-77, 2008.

[2] W.-D. Shi, P.-P. Zou, D.-S. Zhang, and L. Zhou, "Unsteady flow pressure fluctuation of high-specific-speed mixed-flow pump," Transactions of the Chinese Society of Agricultural Engineering, vol. 27, no. 4, pp. 145-152, 2011.

[3] Y.-L. Wu, S.-H. Liu, H.-S. Dou, S.-F. Wu, and T.-J. Chen, "Numerical prediction and similarity study of pressure fluctuation in a prototype Kaplan turbine and the model turbine," Computers and Fluids, vol. 56, pp. 128-142, 2012.

[4] J. Shi, X. Li, and S.-P. Wang, "Dynamic pressure gradient model of axial piston pump and parameters optimization," Mathematical Problems in Engineering, vol. 2014, Article ID 352981, 10 pages, 2014.

[5] J.-T. Liu, S.-H. Liu, Y.-K. Sun, Y.-L. Wu, and L.-Q. Wang, "Prediction of pressure fluctuation of a pump-turbine at noload opening," Journal of Engineering Thermophysics, vol. 33, no. 3, pp. 411-414, 2012.

[6] R. Spence and J. Amaral-Teixeira, "Investigation into pressure pulsations in a centrifugal pump using numerical methods supported by industrial tests," Computers and Fluids, vol. 37, no. 6, pp. 690-704, 2008.

[7] G. Pavesi, G. Cavazzini, and G. Ardizzon, "Time-frequency characterization of the unsteady phenomena in a centrifugal pump," International Journal of Heat and Fluid Flow, vol. 29, no. 5, pp. 1527-1540, 2008.

[8] A. E. Khalifa, A. M. Al-Qutub, and R. Ben-Mansour, "Study of pressure fluctuations and induced vibration at blade-passing frequency of a double volute pump," Arabian Journal for Science and Engineering, vol. 36, no. 7, pp. 1333-1345, 2011. 
[9] F.-J. Wang, L.-X. Qu, L.-Y. He, and J.-Y. Gao, "Evaluation of flow-induced dynamic stress and vibration of volute casing for a large-scale double-suction centrifugal pump," Mathematical Problems in Engineering, vol. 2013, Article ID 764812, 9 pages, 2013.

[10] R. Barrio, J. Parrondo, and E. Blanco, "Numerical analysis of the unsteady flow in the near-tongue region in a volute-type centrifugal pump for different operating points," Computers \& Fluids, vol. 39, no. 5, pp. 859-870, 2010.

[11] Z. Wang, G. Peng, L. Zhou, and D. Hu, "Hydraulic performance of a large slanted axial-flow pump," Engineering Computations, vol. 27, no. 2, pp. 243-256, 2010.

[12] R. Zhu, H. Yan, Q. Fu, and A. Yang, "Numerical calculation of characteristics of tubular pump internal pressure pulsation," Journal of Hydroelectric Engineering, vol. 31, no. 1, pp. 220-225, 2012.

[13] F. Yang and C. Liu, "The flow simulation and experimental study of a large low-head mixed-flow pumping system," ISRN Mechanical Engineering, vol. 2013, Article ID 128326, 12 pages, 2013. 


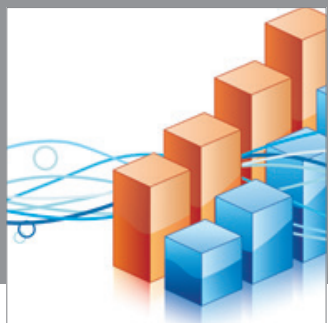

Advances in

Operations Research

mansans

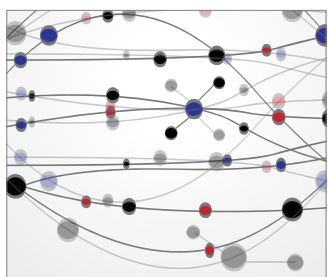

The Scientific World Journal
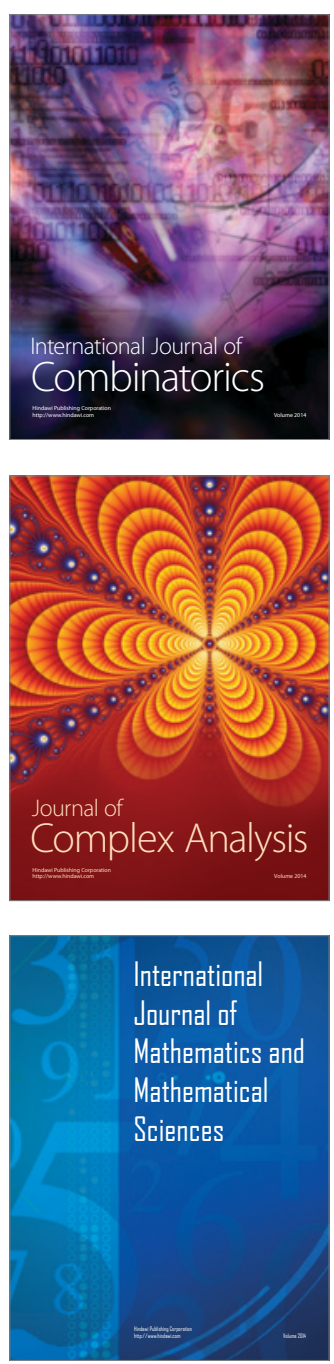
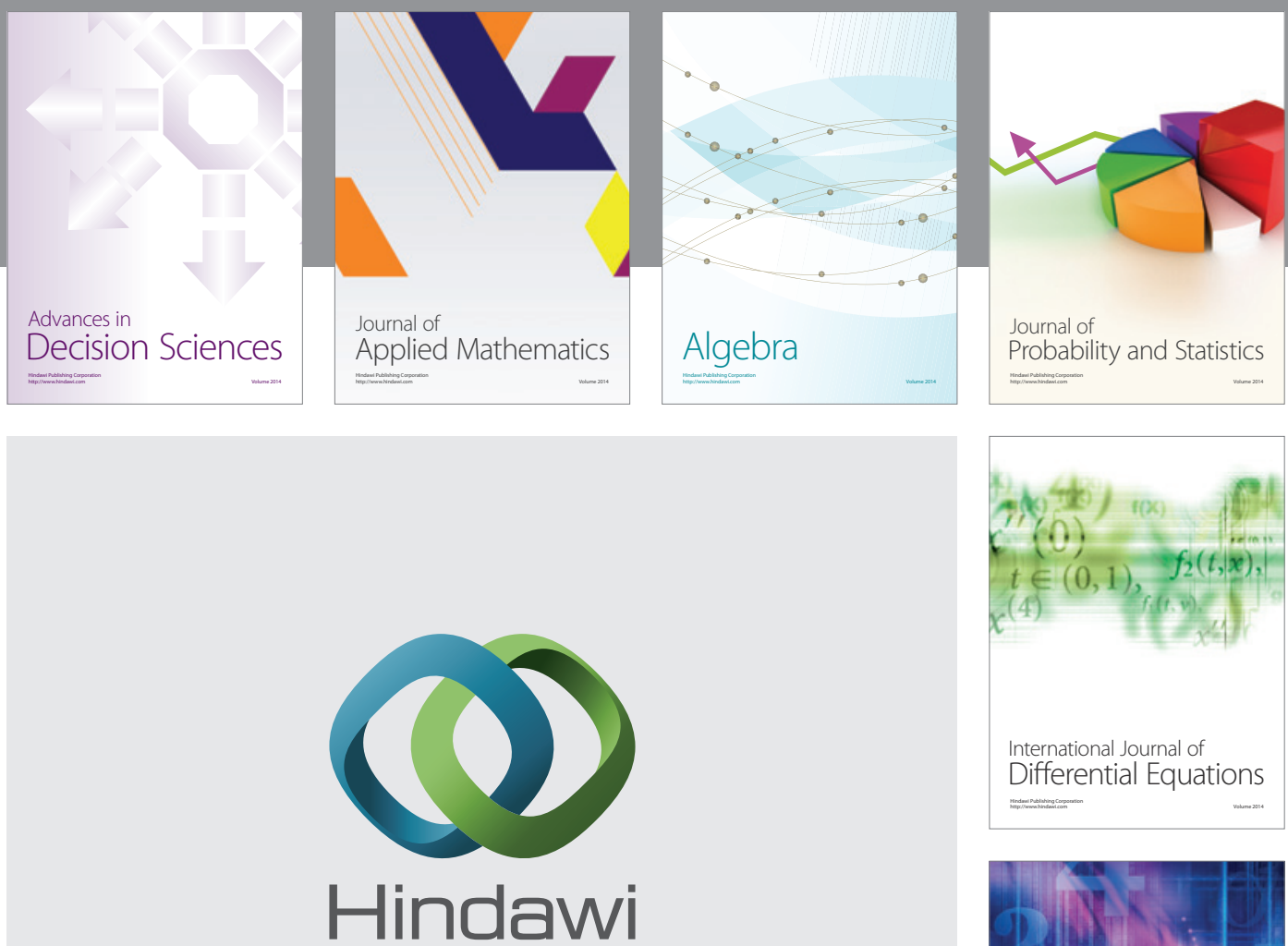

Submit your manuscripts at http://www.hindawi.com
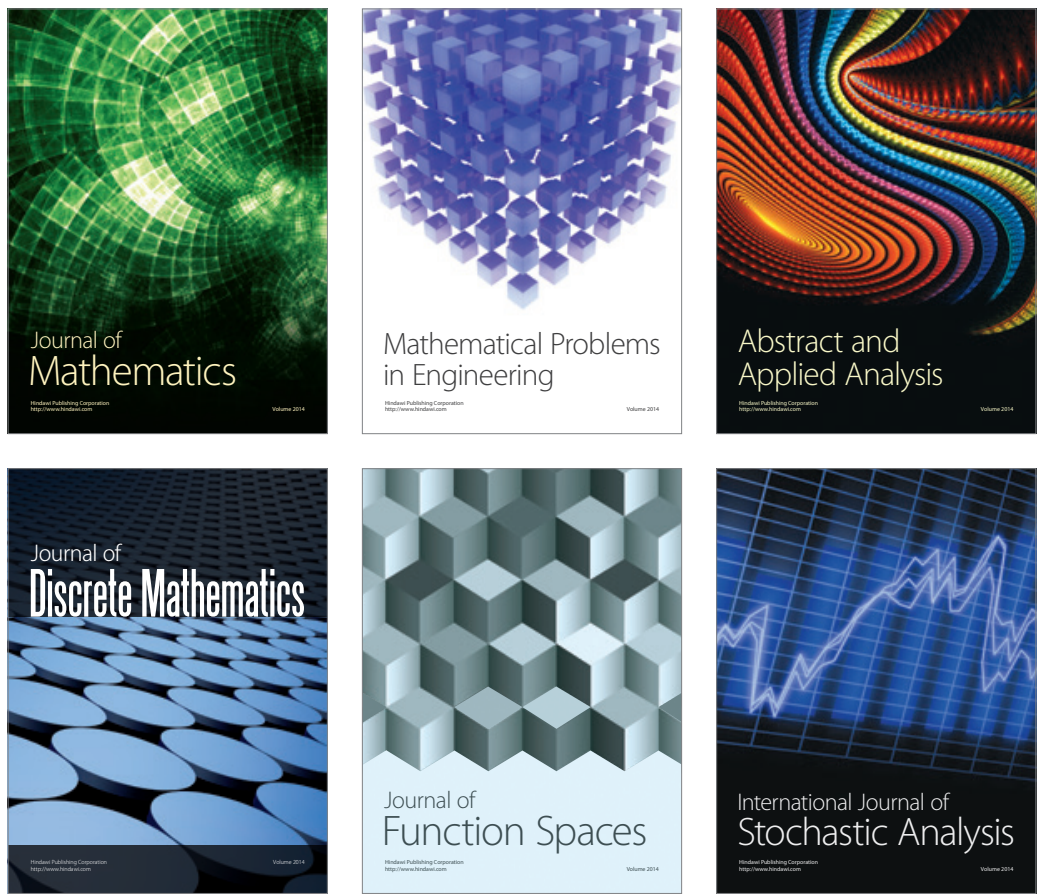

Journal of

Function Spaces

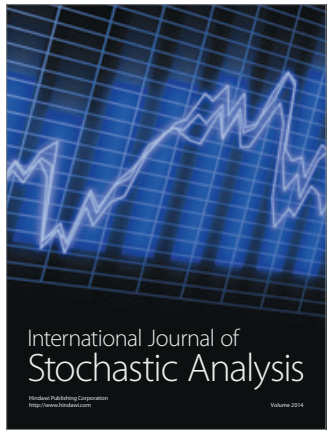

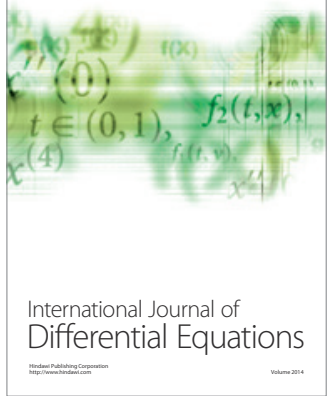
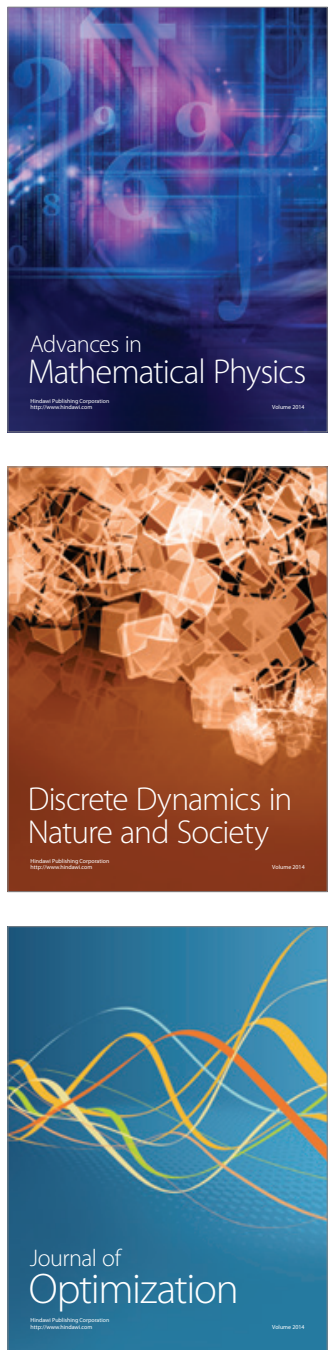